\title{
Cavity polariton condensate in a disordered environment
}

\author{
Martin Thunert, ${ }^{1}$ Alexander Janot, ${ }^{2}$ Helena Franke, ${ }^{1}$ Chris Sturm, ${ }^{1}$ Tom Michalsky, ${ }^{1}$ María Dolores Martín, ${ }^{3,4}$ Luis Viña, $, 3,5$ \\ Bernd Rosenow, ${ }^{2}$ Marius Grundmann, ${ }^{1}$ and Rüdiger Schmidt-Grund ${ }^{1}$ \\ ${ }^{1}$ Institut für Experimentelle Physik II, Universität Leipzig, 04103 Leipzig, Germany \\ ${ }^{2}$ Institut für Theoretische Physik, Universität Leipzig, 04103 Leipzig, Germany \\ ${ }^{3}$ Departamento de Física de Materiales, Universidad Autónoma de Madrid, Madrid 28049, Spain \\ ${ }^{4}$ Instituto de Ciencia de Materiales "Nicolás Cabrera," Universidad Autónoma de Madrid, Madrid 28049, Spain \\ ${ }^{5}$ Instituto de Física de la Materia Condensada, Universidad Autónoma de Madrid, Madrid 28049, Spain
}

(Received 22 January 2015; revised manuscript received 17 November 2015; published 9 February 2016)

\begin{abstract}
We report on the influence of disorder on an exciton-polariton condensate in a ZnO-based bulk planar microcavity and compare experimental results with a theoretical model for a nonequilibrium condensate. Experimentally, we detect intensity fluctuations within the far-field emission pattern even at high condensate densities, which indicates a significant impact of disorder. We show that these effects rely on the driven dissipative nature of the condensate and argue that they can be accounted for by spatial phase inhomogeneities induced by disorder, which occur even for increasing condensate densities realized in the regime of high excitation power. Thus, nonequilibrium effects strongly suppress the stabilization of the condensate against disorder, contrary to what is expected for equilibrium condensates in the high-density limit. Numerical simulations based on our theoretical model reproduce the experimental data.
\end{abstract}

DOI: 10.1103/PhysRevB.93.064203

\section{INTRODUCTION}

The observation of a macroscopically coherent quantum state of exciton-polaritons, a so-called polariton Bose-Einstein condensate (BEC) [1,2], has opened an active and challenging research field. Exciton-polaritons (for brevity, polaritons) are mixed light-matter excitations in a microcavity (MC) $[3,4]$. At finite quasiparticle density, several fascinating phenomena like superfluidity [5-7] and the formation of quantum vortices [8] were discovered. This allows for numerous novel applications like optical parametric oscillators [9], polariton lasers [10,11], and logical elements [12-16], which are usually restricted to low temperatures. However, polariton BECs, even at room temperature, were observed in MCs based on wide-band-gap materials like GaN [17-19] and $\mathrm{ZnO}$ [20-22] and organic materials [23], paving the way for technological applications. At the moment, experiments in these materials are significantly affected by disorder [17,24,25], and a thorough understanding of the impact disorder has on experimental observables in a polariton BEC is called for.

In contrast to conventional BECs, occurring, for example, in cold-atom systems, polaritons have a finite lifetime, which gives rise to unique properties of the condensate. Nonetheless, there remain similarities; for instance, in the absence of disorder, quasi-long-range order of a two-dimensional polariton condensate [26-29] and superfluidity are theoretically expected [30,31] and experimentally observed [5-7]. However, recent theoretical studies have revealed exciting differences between equilibrium and nonequilibrium condensates [32-36]. For example, it is predicted that correlation functions for the condensate wave function decay exponentially [35] and that superfluidity vanishes in the presence of disorder [36].

A polariton BEC is a steady state out of equilibrium, where losses are compensated by external excitation. In the presence of disorder, spatial inhomogeneities of the condensate phase are induced [36]. If the phase fluctuates on length scales comparable to the condensate size, spatial correlations and phase rigidity are strongly reduced. In our work we will show that this leads to significant traces of disorder in the experimentally observed $k$-space intensity distribution and theoretically demonstrate that the ratio of the condensate correlation length to the condensate size is independent of the condensate density. Consequently, in polariton condensates the stabilization against disorder fluctuations with increasing condensate density is strongly suppressed compared to that of condensates in equilibrium.

This prediction is supported by experimental investigations of the impact of disorder on a two-dimensional polariton $\mathrm{BEC}$ in a $\mathrm{ZnO}$-based $\mathrm{MC}$. We measure the $k$-space intensity distribution as a function of excitation power, or, rather, condensate density, and observe significant disorder effects even at high densities. Numerical simulations allow us to make a comparison with experimental data, confirming our theoretical predictions.

For an equilibrium BEC our observations would be unexpected since an increasing density screens the disorder potential and leads to an ordered superfluid state [37-39]. Analogously, for a polariton BEC, interactions also can lead to superfluidity, as observed in clean samples [5-7]. However, as mentioned above, in the presence of disorder the polariton BEC is, strictly speaking, not a superfluid, and long-range order is destroyed [36]. Thus, we expect and observe that disorder affects a dissipative polariton BEC much more than an equilibrium one. Several further observations found in the literature seem to support this. For example, in one-dimensional CdTe MCs [40,41] and ZnO MCs [25] the spatial first-order correlation function of polariton BEC emission was analyzed in the presence of disorder, and significant changes due to disorder were found. In the CdTe MCs the disorder effects remain present even with increasing excitation power, similar to our findings in two-dimensional $\mathrm{ZnO}$ MCs. We note that the correlation length of the assumed disorder potential discussed in Ref. [41] is of the order of microns, which enables the trapping of the entire condensate. This is explicitly excluded 
in our model since the disorder correlation length is assumed to be much smaller than the condensate size, leading to spatial density and phase fluctuations of the condensate instead. Moreover, in various works on two-dimensional polariton BECs in CdTe-based MCs disorder effects were also observed, leading to fluctuations within the far-field photoluminescence (PL) distribution [42] or the spatial first-order correlation function [1]. Even frequency desynchronization between spatially separated condensate fragments can be induced if the ratio between the disorder potential and the polariton interaction potential strength exceeds a critical value [43-46]. However, the dependence of the condensate density on the disorder effects was not analyzed within these works.

This paper is organized as follows: In Sec. II we introduce our theoretical model. We discuss the disorder impact on homogeneously and inhomogeneously excited condensates for quasiequilibrium (weak gain and loss) and driven dissipative (strong gain and loss) condensates, respectively. Furthermore, we provide a general argument that explains our experimental findings. These are presented in Sec. III. In Sec. IV the theoretical predictions are confirmed by comparing experimental data to theoretical simulations. The summary and conclusion can be found in Sec. V.

\section{THEORETICAL PREDICTIONS}

\section{A. Model}

A phenomenological description of the dynamics of the polariton condensate wave function $\Psi(\vec{x}, t)$ is given by an extended Gross-Pitaevskii equation (eGPE) $[47,48]$

$$
\begin{aligned}
i \hbar \partial_{t} \Psi= & \left(-\frac{\hbar^{2}}{2 m} \vec{\nabla}^{2}+V(\vec{x})+U|\Psi|^{2}\right) \Psi \\
& +i\left(R(\vec{x})-\Gamma|\Psi|^{2}\right) \Psi,
\end{aligned}
$$

where $m$ is the effective mass of the lower polariton branch, $V$ is an external potential, and $U>0$ is an on-site interaction constant. The function $R(\vec{x})$ describes the linear part of gain and loss due to inscattering from a reservoir of noncondensed polaritons and the finite lifetime of the condensate. The nonlinearity $\Gamma|\Psi|^{2}$ implements a density-dependent gain saturation, with $\Gamma$ being a gain depletion constant. Since the propagation of the reservoir polaritons can be neglected, the spatial shape of $R(\vec{x})$ can be related to the Gaussian profile of the excitation laser, namely,

$$
R(\vec{x})=\hbar \gamma_{\mathrm{c}}\left(\frac{P}{P_{\mathrm{th}}} e^{-\vec{x}^{2} / \xi_{P}^{2}}-1\right) .
$$

The parameter $\gamma_{c}$ is the condensate decay rate (inverse lifetime $\left.\gamma_{\mathrm{c}}=1 / \tau\right)$. The ratio $P / P_{\mathrm{th}}$ is the excitation power versus its value at the threshold $P_{\text {th }}$ at which condensation is observed first, and $\xi_{P}$ is the waist size of the Gaussian pump spot. We note that for the case of a spatially homogeneous excitation the eGPE (1) was successfully used to analyze a driven dissipative condensate $[32,35]$.

Because of interactions, the condensate energy is blueshifted by $n_{0} U$, where $n_{0}$ is the mean condensate density determined by the balance of gain and loss [for a definition of $n_{0}$ see Eq. (C4)]. The healing length $\xi \equiv \hbar / \sqrt{2 m n_{0} U}$ is obtained by comparing the kinetic and interaction energies of Eq. (1).

The disordered environment is described by a random potential $V(\vec{x})$. We choose Gaussian-distributed $\delta$-correlated disorder with zero mean and variance $\xi_{V}^{2} V_{0}^{2}$; see Appendix C for details. We introduce an effective dimensionless disorder parameter,

$$
\kappa \equiv \frac{\xi_{V} V_{0}}{\xi n_{0} U} .
$$

An analysis of the gain and loss terms in Eq. (1) allows us to define a "nonequilibrium parameter"

$$
\alpha \equiv \frac{\Gamma}{U} .
$$

Its magnitude parametrizes the influence of gain and loss on the polariton BEC. For example, in the limit $\alpha \rightarrow 0$ (keeping $n_{0}$ finite) the equilibrium mean-field description of a BEC is obtained, and on the other hand, in the limit $\alpha \rightarrow \infty$ the condensate is totally dominated by gain and loss.

In this work, we will focus on single-mode steadystate solutions and therefore make the ansatz $\Psi(\vec{x}, t)=$ $\Psi(\vec{x}) \exp (-i \omega t)$, where $\hbar \omega$ is the condensate energy. However, in experimental realizations more than one condensate mode can exist. For any further details we refer to Appendix C.

\section{B. Disorder effects}

\section{Infinite condensate size}

Before we discuss a finite-size polariton BEC we would like to consider a homogeneously excited condensate $\left(\xi_{P} \rightarrow \infty\right)$, such that the reservoir function (2) is a constant in space. We will (i) review disorder effects on an equilibrium condensate $[37,38]$ and (ii) describe differences to a polariton BEC (driven dissipative condensate) [36].

(i) Equilibrium condensate. The disorder potential attempts to pin the condensate into its minima, whereby the energy costs for density deformations [kinetic term in Eq. (1)] have to be compensated. The balance of pinning and kinetic energy determines the density Larkin length $\mathcal{L}_{\mathrm{n}} \approx \sqrt{\pi} \hbar^{2} / m \xi_{V} V_{0}$ $[37,38,49]$. On the other hand, for a sufficiently large interaction energy $n_{0} U$ the disorder gets screened [38]. The ratio of healing to Larkin length, $\xi / \mathcal{L}_{\mathrm{n}} \sim \kappa$, describes this competition of disorder and interaction. For $\xi \ll \mathcal{L}_{\mathrm{n}}\left(\xi \gg \mathcal{L}_{\mathrm{n}}\right)$ the interaction energy is large (small) compared to the disorder potential. Due to the fact that the interaction energy increases with increasing density (and $\left.\xi \propto 1 / \sqrt{n_{0}}\right), \xi / \mathcal{L}_{\mathrm{n}}$ decreases with increasing density, and disorder effects will fade away in this limit. Thus, for sufficiently high densities an equilibrium condensate will be ordered and superfluid [38].

(ii) Nonequilibrium condensate. In a driven system the mean density $n_{0}$ of the condensate is determined by a balance of gain and loss. Disorder induces density fluctuations about this mean value. In a region with reduced density, compared to $n_{0}$, the gain mechanism tries to compensate the depletion, and more particles are scattered into the condensate than decay. On the other hand, in a region with increased density more particles decay than are injected from the reservoir. By virtue of the continuity equation, these local particle sources and sinks are connected by condensate currents. Because the density 
fluctuates randomly in space, a random distribution of sources and sinks forms, and thus, a random pattern of current flow is generated. The condensate current is proportional to the product of the density and the gradient of the condensate phase. Since the current is not constant, the phase cannot vary uniformly in space, and thus, a random current configuration gives rise to a spatially fluctuating phase. We note that in this work the term "fluctuations" will be used for random spatial inhomogeneities. The correlation length, over which the phase typically varies by $2 \pi$, is given by $\mathcal{L}_{\phi} \approx \sqrt{2} \pi \mathcal{L}_{\mathrm{n}} / \alpha$. This scale can be obtained by a generalized Imry-Ma argument [36]: a condensate current flowing out of (into) a region of diameter $\mathcal{L}_{\phi}$ is generated by an effective source (sink) determined through an area average of multiple random sources and sinks. In contrast to an equilibrium condensate $\left(\alpha \rightarrow 0\right.$ with $\mathcal{L}_{\phi} \rightarrow \infty$ ), the phase fluctuations occurring in the case $\mathcal{L}_{\phi}<\infty$ destroy the quasi-long-range order of the condensate. As a consequence of these phase fluctuations, the superfluid stiffness vanishes in the thermodynamic limit even for weak disorder, and a superfluid behavior is only present below a finite length scale, namely, the superfluid depletion length $\mathcal{L}_{s} \approx \sqrt{2} \pi \mathcal{L}_{\mathrm{n}} / \alpha^{2}[36]$.

\section{Finite condensate size}

From the analysis above we conclude that in a disordered environment a condensate of size $L_{c} \ll \mathcal{L}_{\phi}$ will behave completely different from one of size $L_{c} \sim \mathcal{L}_{\phi}$. In the following, we discuss these two scenarios sketched schematically in Fig. 1(b).

For scenario I with $L_{c} \ll \mathcal{L}_{\phi}$ (called quasiequilibrium in the following) the phase is correlated over the entire condensate region, and disorder induces mainly density fluctuations. As discussed above, the impact of disorder will decrease with increasing density, which should be directly observable by increasing the excitation power. Such a percolation transition from a disordered regime to an ordered regime was predicted (for a polariton BEC in equilibrium) in Ref. [39].

In the presence of gain and loss disorder induces phase fluctuations, as explained above. For scenario II we assume that the phase correlation length $\mathcal{L}_{\phi}$ is comparable to the condensate size $L_{c}$, i.e., $\mathcal{L}_{\phi} \sim L_{c}$, such that spatial correlations and superfluidity are destroyed. The ratio $L_{c} / \mathcal{L}_{\phi} \propto$ $\left(V_{0} \xi_{\mathrm{P}} \xi_{\mathrm{v}} m / \hbar^{2}\right)(\Gamma / U)$ does not depend on the condensate density and thus is independent of the excitation power. A similar conclusion holds for the ratio $L_{c} / \mathcal{L}_{s}$. As a consequence, a condensate stabilization with increasing density, as present in an equilibrium system, is strongly suppressed.

In order to make our analysis more quantitative, we have studied theoretically the excitation power dependence of the two-dimensional $k$-space intensity $I_{P}(\vec{k}) \propto \gamma_{c}\left|\Psi_{\vec{k}}\right|^{2}$, which can be directly compared to experimental data. To this end, Eq. (1) was simulated for many disorder realizations (see Appendix D for details). We have extracted the expectation value, denoted by $\mu_{P}(k)$, and the variance, denoted by $\sigma_{P}^{2}(k)$, of the normalized intensity $I_{P}(\vec{k})$ by averaging over disorder configurations. We note that for a sufficiently large number of realizations, the disorder average restores radial symmetry, such that the expectation values $\mu_{P}(k)$ and $\sigma_{P}^{2}(k)$ depend on the magnitude $k=|\vec{k}|$ of only the wave vector. (a) low condensate density

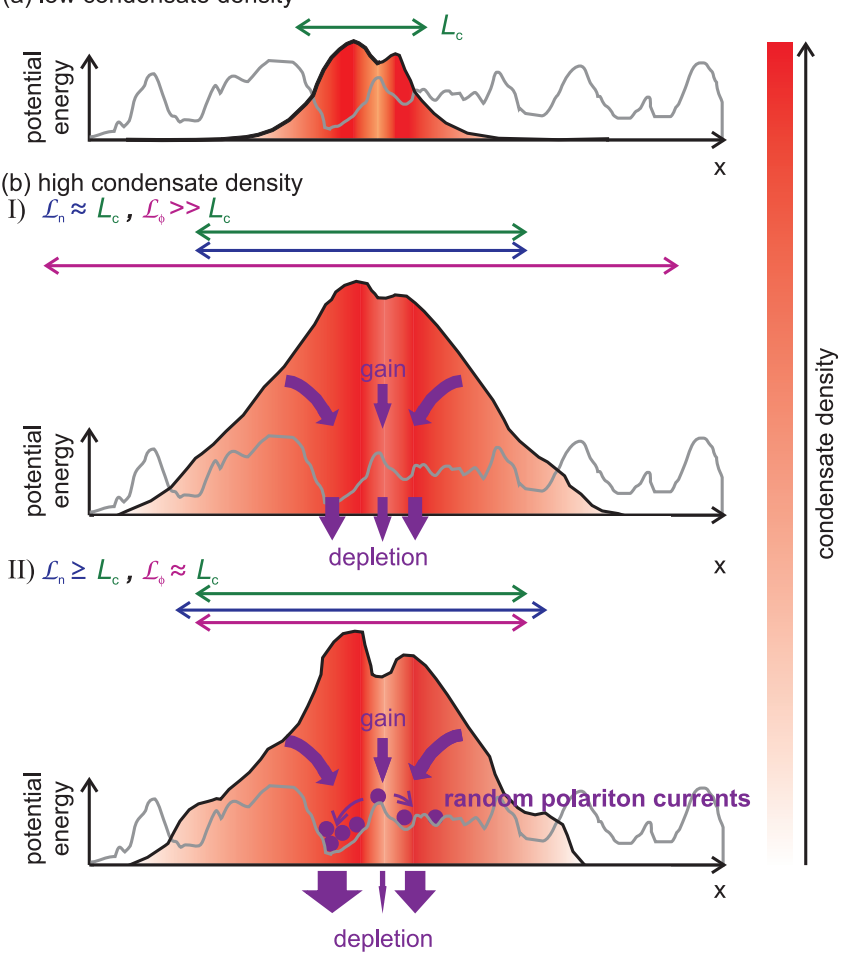

FIG. 1. Disorder impact on a polariton condensate for increasing density. The gray lines represent the disorder potential. The condensate density is depicted by a red color code, and the corresponding interaction potential is shown by a black line. In all cases, a Gaussian-shaped excitation spot is assumed. (a) For low condensate densities significant disorder effects are present. (b) Depending on the nonequilibrium nature of the condensate two different scenarios are expected for a high density. Scenario I (quasiequilibrium condensate): disorder induces density fluctuations $\left(\mathcal{L}_{\mathrm{n}} \sim L_{c}\right.$, where $L_{c}$ is condensate size), whereas the condensate phase remains unperturbed $\left(\mathcal{L}_{\phi} \gg L_{c}\right)$. For sufficiently high densities the interaction potential screens the disorder, which results in a weakly perturbed condensate. Scenario II (driven dissipative condensate): the presence of disorder in combination with gain and loss leads to phase fluctuations $\left(\mathcal{L}_{\phi} \sim L_{c}\right)$. These are density independent, and thus disorder effects persist with increasing density.

In Fig. 2, the results for $\mu_{P}$ and $\sigma_{P}^{2}$ are shown for scenarios I (left panels) and II (right panels). We find that the intensity $I_{P}$ vanishes for all wave vectors outside of the lower-polariton dispersion $\left(k \gtrsim \xi^{-1}\right)$ and that its average value does not change qualitatively compared to a disorder-free system (cf. Ref. [50]). However, for a single snapshot (see Fig. 5) disorder breaks the radial symmetry and induces intensity fluctuations proportional to $\sigma_{P}$. For scenario I and for wave vectors $|k| \lesssim$ $\xi^{-1}$, these fluctuations decay linearly with inverse excitation power, in agreement with the expectation $\sigma_{P}^{2} \sim \kappa^{2} \propto 1 / P$ for $\kappa \ll 1$. We note that regions with $k \approx \xi^{-1}$ show a high ratio $\sigma_{P} / \sigma_{P_{0}}$ (peaks in Fig. 2, bottom left panel). In this $k$ region, the emission intensity is increasing very rapidly with excitation power (see Fig. 2, top left panel) because of the repulsive potential hill created by the finite excitation spot [50]. Thus, the increase of fluctuation strengths with excitation power for $k \approx \xi^{-1}$ is really due to the increase of emission power and 

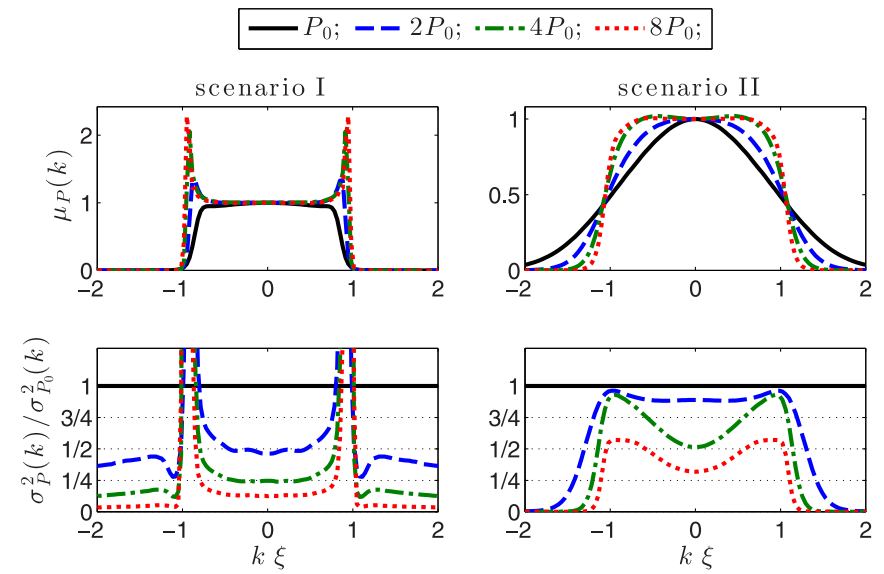

FIG. 2. Expectation value $\mu_{P}$ (top row) and variance $\sigma_{P}^{2}$ (bottom row) of the (normalized) intensity distribution $I_{P}(k)$. In order to compare the fluctuations for increasing excitation powers we present the ratio $\sigma_{P}^{2}(k) / \sigma_{P_{0}}^{2}(k)$, with $P_{0}=4 P_{\text {th }}$. The left and right columns depict a quasiequilibrium (scenario I) and a driven dissipative (scenario II) condensate, respectively. For wave vectors $|k \xi| \lesssim 1$, scenario I shows a linear reduction of fluctuations with inverse excitation power, $\sigma_{P}^{2}(k) \propto 1 / P$, while scenario II exhibits a suppressed stabilization with increasing excitation power. We averaged 1560 disorder realizations and used $\mathcal{L}_{\mathrm{n}} / L_{c}=1(4.5), \mathcal{L}_{\phi} / L_{c}=10$ (2.5), $\mathcal{L}_{s} / L_{c}=20(0.3)$ for scenario I (II).

does not yield information about the screening of the disorder potential for high-condensate densities.

For scenario II, the stabilization with increasing excitation power is suppressed (see bottom right panel of Fig. 2). Compared to scenario I, the decrease of $\sigma_{P}^{2}$ with increasing condensate density is weaker than $\sigma_{P}^{2} \propto 1 / P$. These findings agree well with our argument provided above.

The reservoir of noncondensed polaritons interacts with the condensate and thus leads to an increase of the blueshift $[47,51,52]$. Usually, this is accounted for by adding a potential term proportional to the reservoir density in Eq. (1) [47]. Such a term will modify the emission frequency of the condensate [real part of Eq. (1)]; however, it does not change the nonequilibrium continuity equation [imaginary part of Eq. (1)]. Hence, the mechanism of generating random condensate currents is not altered qualitatively by reservoir-condensate interaction, and thus, we believe that they can be safely neglected for our analysis.

\section{EXPERIMENT}

In this section we discuss the experimentally observed behavior of the far-field PL emission pattern of a polariton condensate in a $\mathrm{ZnO}$-based $\mathrm{MC}$ with pronounced structural disorder as a function of excitation power. For this experiment, the sample was excited using a pulsed $\mathrm{Nd}$ :YAG laser with a pulse duration of $500 \mathrm{ps}$. This is three orders of magnitude larger than the polariton relaxation time $(0.4 \mathrm{ps})$ which is determined from the spectral linewidth of the condensate emission. Thus, we can assume a quasi-continuous-wave excitation, which justifies the comparison with numerical simulations based on a steady-state theory, which will be discussed in Sec. IV. Further details about the experimental
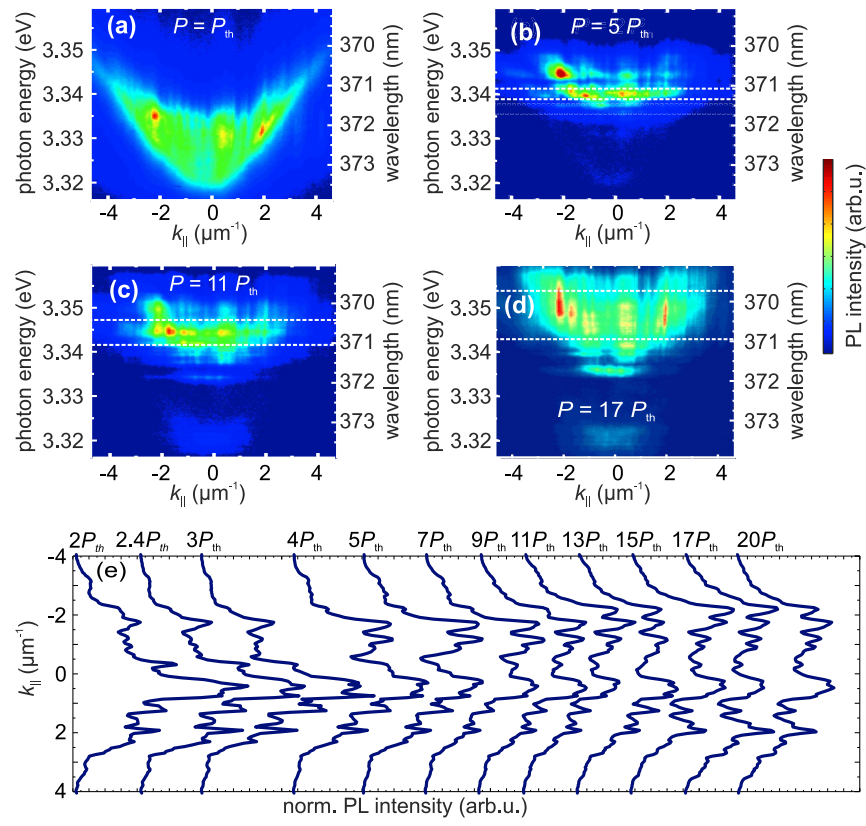

FIG. 3. (a)-(d) Excitation power series of the far-field PL emission in a linear false-color scale for $T=10 \mathrm{~K}$ and a detuning of $\Delta=$ $-30 \mathrm{meV}$. The excitation power is normalized to the condensation threshold $P_{\text {th }}$. (e) $I_{P}(k)$ profiles of the condensate are extracted. These are integrated over the energy range $\Delta E$ marked by the white lines in the far-field emission pattern. The PL intensity for each power is normalized to the mean value of each $I_{P}(k)$ profile.

setup can be found in Appendix A. The MC consists of a half-wavelength $\mathrm{ZnO}$ cavity, which simultaneously acts as an active medium, showing a quality factor of about 1000 and a maximum coupling strength of about $45 \mathrm{meV}\left(\Omega_{\text {Rabi }} \approx\right.$ $90 \mathrm{meV}$ ) at $T=10 \mathrm{~K}$. By using a wedge-shaped cavity, the detuning between the cavity mode energy and the excitonic transition energy strongly varies with the lateral sample position. Structural investigations (atomic force microscopy, $\mathrm{x}$-ray diffraction, cross-sectional transmission electron microscopy) yield a smooth but polycrystalline cavity layer, exhibiting a low interface roughness of $R_{\mathrm{rms}}=1.9 \mathrm{~nm}$. Furthermore, the cavity layer is preferentially $c$-plane oriented and laterally textured, containing large grains aligned in the growth direction reaching from the bottom to the top (grain sizes ranging from 20 up to $120 \mathrm{~nm}$ ). Further information about the sample properties can be found in Ref. [24]. Due to the textured structure we suppose that an electronic disorder potential is primarily caused by depletion of carriers, e.g., aluminum donor bound excitons [53], due to interface band bending at grain boundaries [54] (see Appendix B for details).

Figures 3(a)-3(d) show the excitation power dependence of the PL $k$-space emission pattern for $T=10 \mathrm{~K}$ and detuning $\Delta=-30 \mathrm{meV}$. We deduce a polariton effective mass of $m=4.4 \times 10^{-5} m_{\mathrm{e}}$ ( $m_{\mathrm{e}}$ is the free-electron mass) from the dispersion of the lower polariton branch (LPB; not shown here). The excitation power density at condensation threshold is $P_{\text {th }}=79 \mathrm{~W} \mathrm{~cm}{ }^{-2}$. Note that the determination of the excitation power density at threshold is quite complex, e.g., due to the coexistence of intense emission from uncondensed polaritons for $P \gtrsim P_{\mathrm{th}}$, but significant for the comparison with 
theoretical calculations discussed in Sec. IV. Details of the experimental determination of $P_{\text {th }}$ can be found in Sec. I of the Supplemental Material [55].

In all cases investigated here, the condensate emission is distributed dispersionless at horizontal lines in $k$ space with maximum intensity between the LPB dispersion, which is visible in the far-field PL images (see Fig. 3) for low excitation power $P \gtrsim P_{\text {th }}$. This indicates a weak expansion of the condensed polaritons due to the background potential induced by the excitation spot, whose size is similar or even larger than the polariton propagation length [24,50]. For the lowest excitation power shown here, $P=P_{\text {th }}$, the emission intensities from the uncondensed polaritons and the condensate are of same order, which prevents a clear distinction. With increasing excitation power the BEC-states undergo a blueshift due to the increasing interaction potential, and we observe several states with different energies. Previous studies in the literature on this multimode behavior show that the emission from coexisting individual modes originates from different regions of the same condensate $[43,44,56]$. However, other studies on polariton condensates in a disordered environment found that long-range spatial coherence is still present for their energy-averaged emission [1,42], indicating persistent correlations between different, possibly spatially separated, condensate states.

For a wide range of excitation powers, condensate emission out of two energy ranges is observed, which are stable and energetically well separated. For further analysis we select only one of these energy channels in order to compare with numeric simulations of a single-mode condensate (see Sec. IV). In Figs. 3(b)-3(d) we mark the selected energy channel by two white dashed lines. This delimitation is defined by an energy range $\Delta E$ which corresponds to the excitation-power-dependent full width at half maximum (FWHM) of the condensate emission. Figure 3(e) shows the far-field emission profiles $I_{P}(k)$ for the selected energy channel and increasing excitation power, integrated over $\Delta E$.

The $I_{P}(k)$ profiles show several randomly distributed inhomogeneities and differ strongly from the smooth and ideally radial symmetric distribution expected for a disorder-free sample [50]. Remarkably, the intensity fluctuations persist even for high excitation power, i.e., high condensate densities. We note that the constant sharp stripes in the $I_{P}(k)$ profile at a specified $k$ for all excitation powers are caused by imperfections of the setup, probably due to the microscope objective.

A similar finding with increasing excitation power was also observed for other detunings $\Delta=-50 \mathrm{meV}, \ldots,-10 \mathrm{meV}$, and we conclude that our observation does not depend significantly on the particular choice of detuning within the mentioned range.

To investigate the temporal coherence properties of the condensate we used a Michelson interferometer in the planemirror (PM)-retroreflector (RR) configuration to superimpose the PL emission of polaritons with opposite emission angles, or, rather, wave vectors [57]. For this experiment, the sample was excited by a frequency-tripled Ti:sapphire laser at 266 $\mathrm{nm}$ with a pulse duration of about 2 ps. Further details of the setup are provided in Appendix A. The RR is mounted on a motorized linear stage that allows us to vary the path difference $\Delta s$ between the emission collected from both interferometer arms. Figures 4(a) and 4(b) show two selected interferograms
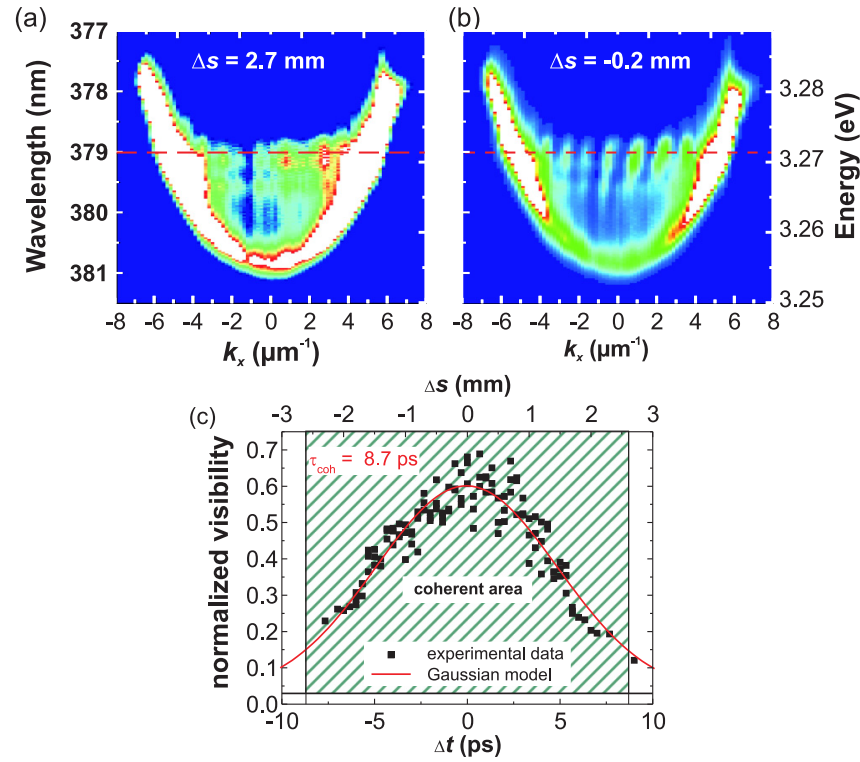

FIG. 4. (a) and (b) Energy-resolved far-field PL interference pattern in a linear false-color scale for $T=10 \mathrm{~K}$ and $\Delta=-47 \mathrm{meV}$. (a) Large path difference $\Delta s$ : uncorrelated far-field PL emission (basically, the sum of the emission from the individual interferometer arms); (b) path difference close to zero: distinct interference fringes indicating mutual temporal coherence of the polariton emission. The red dashed line indicates the energy of the investigated condensate state. (c) Normalized visibility of the interference fringes as a function of the path difference.

of the $I(E, k)$ emission pattern for large [Fig. 4(a)] and small [Fig. 4(b)] path differences $\Delta s$, respectively. To investigate the temporal coherence properties of the polariton condensate we analyzed the normalized visibility of the interference fringes,

$$
V_{\text {norm }}=\frac{I_{\text {interf }}-I_{R R}-I_{P M}}{2 \sqrt{I_{R R} I_{P M}}}=g^{1}(\Delta t) \cos \left(\phi_{12}\right),
$$
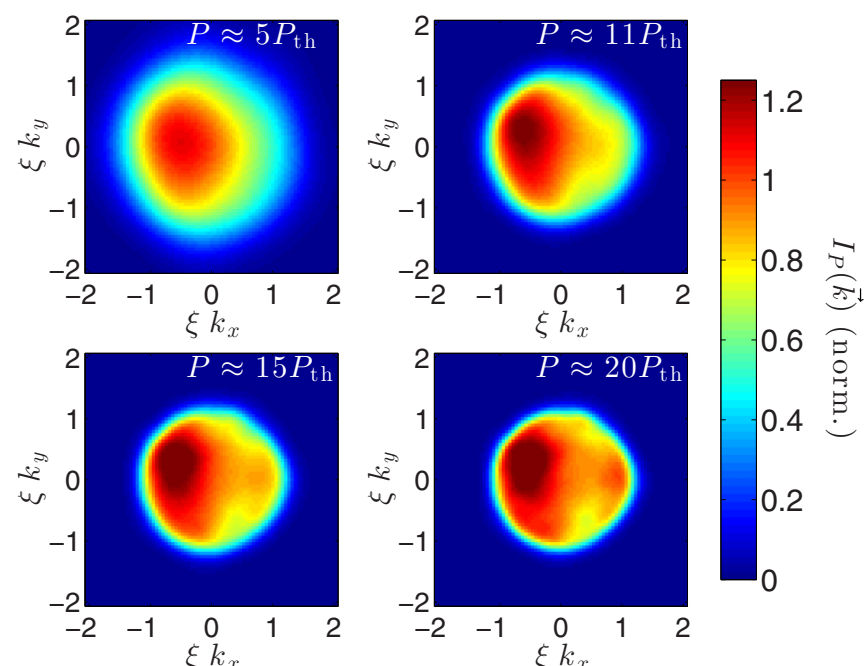

FIG. 5. Snapshots of the normalized two-dimensional intensity distribution $I_{P}(\vec{k})$ of a polariton BEC with $\mathcal{L}_{\phi} \sim L_{c}$ in a disordered environment for increasing excitation power $P$. The parameters used are presented in Table I. 
TABLE I. Parameters extracted from experiment and corresponding parameters used for simulations as well as relevant length scales. For definitions see Appendix C.

\begin{tabular}{lccccc}
\hline \hline$m$ & $\tau$ & $\xi_{\mathrm{P}}$ & $P_{\mathrm{th}}$ & $P / P_{\mathrm{th}}$ & $d \Delta E / d\left(P / P_{\mathrm{th}}\right)$ \\
\hline $4.4 \times 10^{-5} \mathrm{~m}_{\mathrm{e}}$ & $0.4 \mathrm{ps}$ & $2 \mu \mathrm{m}$ & $79 \mathrm{~W} / \mathrm{cm}^{2}$ & $2, \ldots, 20$ & $0.7 \mathrm{meV}$ \\
\hline$\alpha$ & $P / P_{\mathrm{th}}$ & $\xi_{\mathrm{V}} V_{0} / l_{c} \hbar \gamma_{c}$ & $\xi_{\mathrm{P}} / l_{\mathrm{c}}$ & $\mathcal{L}_{\mathrm{n}} / L_{c}$ & $\mathcal{L}_{\phi} / L_{c}$ \\
\hline 7 & $5,11,15,20$ & 0.125 & 3 & 5 & 3 \\
\hline \hline
\end{tabular}

as a function of the temporal delay $\Delta t=\frac{\Delta s}{c}$, where $c$ is the speed of light [see Fig. 4(c)]. Here, $I_{\text {interf }}$ is the intensity of the interference pattern, $I_{\mathrm{RR}}$ and $I_{\mathrm{PM}}$ are the intensities of the $\mathrm{RR}$ and PM arms, respectively, $g^{1}$ is the first-order coherence function, and $\phi_{12}$ is the phase difference between the emissions from the individual interferometer arms. By assuming a Gaussian decay of $g^{1}(\Delta t)=g^{1}(0) \exp \left[-(\pi / 2)\left(\Delta t^{2} / \tau_{\text {coh }}^{2}\right)\right]$ [58] we determined a coherence time of about $\tau_{\text {coh }}=8.7 \mathrm{ps}$. This is more than 50 times larger than the lifetime of the uncondensed polaritons of about $160 \mathrm{fs}$, which is deduced from the spectral linewidth of the polariton emission for $P \leqslant P_{\text {th }}$ and for an energy range similar to the condensate energy at $P=P_{\text {th }}$. Consequently, the coherence of the investigated quantum system is conserved during the multiple reabsorption and reemission processes, which can thus be identified as a condensate. We note that the experimentally estimated coherence time is a lower limit for the real value. We identify two experimental artifacts that restrict the determination of the real condensate's coherence time, namely, a spectrally and path-difference-dependent phase shift $\phi_{12}(\lambda, \Delta s)$ (artifact A) and a fast decay of the condensate emission intensity due to the short excitation pulses of about 2 ps that are used for the coherence-time measurement (artifact B). By analyzing the impact of these artifacts quantitatively (see Supplemental Material, Sec. II), we roughly estimated the expected real values for the coherence times of $\tau_{\mathrm{coh}}^{A}=10.3 \mathrm{ps}$ and $\tau_{\text {coh }}^{B}=14 \mathrm{ps}$. By applying both corrections simultaneously, a maximum coherence time of $\tau_{\text {coh }}=24$ ps was estimated.

For an ideal (homogeneous, disorder-free) condensate a linewidth of $\Delta E \approx 0.66 h / \tau_{\text {coh }}=0.66 h / 8.7 \mathrm{ps}=0.31 \mathrm{meV}$ would be expected for the condensate emission according to the Wiener-Khinchin theorem [58] (and even less assuming the corrected values for $\tau_{\text {coh }}$ ), where $h$ is the Planck constant. This is about a factor of 6.5 smaller than the observed minimum linewidth of $2 \mathrm{meV}$ for the condensate emission in this experiment. Since the investigated condensate is a complex quantum system including spatial density and phase fluctuations, we assume that the Wiener-Khinchin theorem cannot be applied here. We rather suppose that the mechanism which causes a broadening of the emission linewidth (e.g., repulsive particle interaction [59]) does not affect the coherence time to the same extent. This is supported by the quantitative discrepancy between the emission linewidth and the coherence time, which is observed also in a CdTe [1] as well as in a ZnO MC [22]. We note that despite the fast decay of polaritons, condensate emission can be observed up to 90 ps after the arrival of the exciting laser pulse, which thus allows for the experimental observation of coherence in the mentioned time range.

In summary, the experimental observations indicate a strong impact of disorder on the polariton BEC even at high excitation power well above the condensation threshold. As discussed in Sec. II the suppression of disorder effects with increasing condensate density is strongly hindered for a polariton BEC. We assume that the interplay of gain and loss and disorder prevents a stabilization at high excitation power also in the experiment.

\section{COMPARISON BETWEEN THE THEORETICAL MODEL AND EXPERIMENT}

In the following, we will compare our experimental observations with numerical simulations.

At threshold $P=P_{\text {th }}$ a crossover from a noncondensed state to a polariton BEC takes place, typically indicated by a superlinear increase of the emission intensity. Such a transition is not very well described by the eGPE (1). For this reason, the data analysis is done well above threshold, where both experimentally observed and theoretically calculated blueshift (condensate density) increase linearly with pump power. We note that the evolution of the experimentally measured polariton blueshift $\Delta E$ as a function of the excitation power shows two kinks at $P=2 P_{\text {th }}$ and $P=4 P_{\text {th }}$ (see Fig. 7 in Appendix B). We believe that the slope of $\Delta E$ for $P<2 P_{\text {th }}$ is predominantly caused by an electronic disorder potential, which starts to saturate for $P=2 P_{\mathrm{th}}$, and that for $P \geqslant 4 P_{\text {th }}$ the blueshift is governed by condensate-condensate interactions. Further discussions are presented in Appendix B and references therein.

For the comparison between the theoretical model and the experimental data, the parameters of the eGPE (1) are chosen according to the experiment (see Table I) [60]. We note that a quantitative determination of the disorder parameter from experiment is very challenging (see discussion in Appendix B), and we chose $\xi_{\mathrm{V}} V_{0} \approx 0.15 \mu \mathrm{m} \mathrm{meV}$ for simulations.

For a typical disorder realization, a series of numerically obtained snapshots of the two-dimensional intensity distribution $I_{P}(\vec{k})$ for increasing excitation power is shown in Fig. 5. These images correspond to a polariton BEC described by scenario II. We clearly observe a disorder-induced deviation from the ideally radial distribution, which does not converge to a symmetric intensity distribution while increasing the excitation power. Such an asymmetry as well as its persistence is also observed experimentally (see Fig. 3) and thus agrees qualitatively with our simulations. We note that the experimental data represent the intensity distribution of one disordered sample and correspond to a one-dimensional cut along a given line crossing the origin of the two-dimensional $k$-space distribution, for example, the $x$ axis.

For a quantitative analysis we compare directly the experimental measurements with the numerically computed expectation value $\mu_{\mathrm{P}}$ and variance $\sigma_{\mathrm{P}}^{2}$ of the intensity distribution. To this end we symmetrize the experimental data 

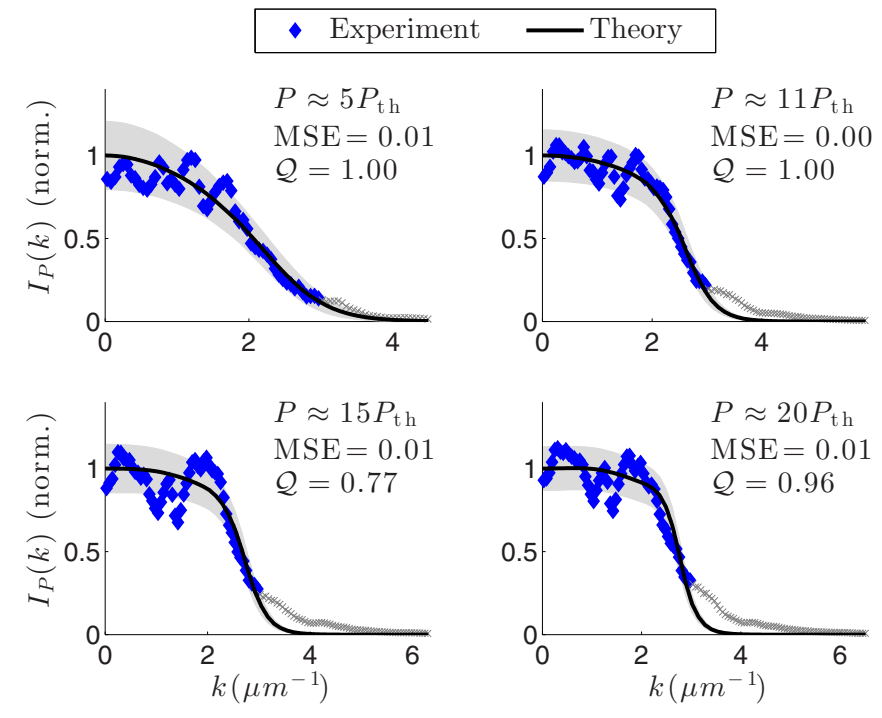

FIG. 6. Experimental $I_{P}(k)$ distribution (blue diamonds) compared to numerical simulations of scenario II for increasing excitation power $P$. The black solid lines depict the expectation value, whereas the gray band indicates the standard deviation. For high momenta we have excluded systematically biased data (gray crosses). For each plot the mean-square error (MSE) and the goodness-of-fit value $\mathcal{Q}$ (see text) are computed. For used parameters see Table I.

$I_{P}(k) \rightarrow\left[I_{P}(k)+I_{P}(-k)\right] / 2$, with $k \geqslant 0$, and superimpose them with the results of the numerical simulations. Since the condensate density and healing length are hard to determine experimentally, we fix the scaling of the $x$ and $y$ axes by a least-squares fit. Figure 6 shows the result. We have excluded experimental data with wave vectors $k \geqslant 3 \mu \mathrm{m}^{-1}$ because a systematic artifact is present for all $k=3, \ldots, 4 \mu \mathrm{m}^{-1}$ and for all excitation powers [61]. In order to quantify the agreement between theory and experiment we introduce the mean-square error (MSE) and the goodness-of-fit value $\mathcal{Q}$ (see Appendix D for definitions). $\mathcal{Q}$ is a probability: if $\mathcal{Q} \sim 1$, the simulations describe the experimental data. On the other hand, if $\mathcal{Q} \ll 1$, the theoretical model does not reproduce the experiment. The experimental data are well described by simulations (of scenario II; see Fig. 6): the MSE is close to zero, and the goodness-of-fit value $\mathcal{Q}$ remains close to 1 for all pump powers studied. In contrast, trying to reproduce the experimental observations by simulations of a polariton BEC described by scenario I (quasiequilibrium) fails (see Fig. 8 in Appendix D). Thereto, we had chosen a nonequilibrium parameter $\alpha=0.5$ and slightly increased the disorder strength. Then, the $\mathcal{Q}$ value dropped from $\mathcal{Q} \approx 10^{-8}$ at $P \approx 5 P_{\text {th }}$ to $\mathcal{Q} \approx 10^{-21}$ at $P=20 P_{\text {th }}$.

\section{SUMMARY AND CONCLUSION}

In this work we have characterized a polariton condensate in a disordered environment. Our theoretical analysis shows that spatial fluctuations of the condensate phase, which are induced by the interplay of disorder and gain and loss of particles, do not depend on the mean condensate density. This leads to a reduced stabilization against disorder fluctuations with increasing density, in contrast to an equilibrium condensate.
To verify our prediction we have analyzed experimentally the photoluminescence emission of a $\mathrm{ZnO}$-based microcavity. Indeed, we find a lack of stabilization with increasing density in terms of pronounced intensity fluctuations within the $k$-space emission pattern even at high excitation power. This experimental finding can be reproduced by numerical simulations. From this we conclude that the polariton condensate in the microcavity is exposed to significant structural disorder and that the persistence of disorder effects even at high excitation power, well above the condensation threshold, relies on the intrinsic nonequilibrium nature of polaritons. We note that these findings may also explain the observation of similar phenomena for polariton condensates in microcavities based on other materials, e.g., CdTe or GaN $[17,43,44]$.

\section{ACKNOWLEDGMENTS}

We acknowledge experimental support and fruitful discussions from the group of N. Grandjean at EPFL. A.J. is supported by the Leipzig School of Natural Sciences BuildMoNa. This work was supported by Deutsche Forschungsgemeinschaft through project GR 1011/20-2, by Deutscher Akademischer Austauschdienst within the project PPP Spain (ID 57050448) and by Spanish MINECO Projects No. MAT2011-22997 and No. MAT2014-53119-C2-1-R.

M.T. and A.J. contributed equally to this work. M.T. performed the experimental part, and A.J. carried out the theoretical analysis.

\section{APPENDIX A: EXPERIMENTAL SETUP}

In order to investigate the optical properties of the polariton condensate, we applied two different photoluminescence configurations, which have in common a nonresonant and pulsed excitation as well as a detection of the far-field emission. The setup to investigate the disorder effects on the polariton distribution and their dynamics as a function of the excitation power is described in Ref. [24]. Here, the excitation was carried out by a pulsed Nd:YAG laser with pulse duration of $500 \mathrm{ps,}$ whose Gaussian excitation spot covers a sample area of about $10 \mu \mathrm{m}^{2}$.

For the coherence measurements, the sample was excited via a frequency-tripled Ti:sapphire laser at $266 \mathrm{~nm}$ (repetition rate of $76 \mathrm{MHz}$, pulse length $\approx 2 \mathrm{ps}$ ). The PL signal of the Fourier plane was sent to a Michelson interferometer in the mirror-retroreflector configuration. The retroreflector image is a centrosymmetric counterpart of the mirror-arm image. In the resulting interferogram we superimposed the signal with wave vector $\overrightarrow{k_{\|}}$with that of $-\vec{k}_{\|}$. Interference maxima occur when the path difference between the individual beams, $\Delta L=c \Delta t$, is an integer multiple of the PL emission wavelength, with $\Delta t$ being the delay between the beams and $c$ being the speed of light. With the help of a streak camera the relative delay between the two arms was set to zero for $k_{\|}=0$.

Real-space measurements with a sufficient spatial resolution could not be performed due to a spherical aberration induced by the cryostat window. For the conditions used in our experiments, namely, the UV spectral range, a window thickness of $1.5 \mathrm{~mm}$, and the large range of collected emission angles of $\pm 23^{\circ}$, the resulting spatial distortion of the image 
is larger than the structural fluctuations that we would like to resolve. Consequently, the distortion of the measured real-space image prevents precise investigation of the spatial distribution of the luminescence as well as spatially resolved correlation measurements. We note that far-field images are not affected by the cryostat window, which causes a parallel beam shift but does not change the angle of the transmitted rays.

\section{APPENDIX B: ORIGIN OF DISORDER POTENTIAL}

Due to the dual light-matter nature of the polaritons, the effective disorder potential can be of photonic as well as electronic origin.

Photonic disorder can be caused by surface and interface roughness as well as thickness fluctuations within the MC structure. This leads to a spatially fluctuating cavity length and therefore to a variation of the cavity photon energy. Due to the results of other $\mathrm{ZnO}$-based $\mathrm{MCs}$, a minimum potential strength of $V_{C} \geqslant 2 \mathrm{meV}$ can be expected [21,62]. The corresponding correlation length $\xi_{V}$ is of the order of the photonic wavelength, of about $370 \mathrm{~nm}$.

In the literature, electronic disorder is usually neglected $[25,40,43,63,64]$. In contrast to this, we assume a strong influence of an electronic background potential caused by randomly distributed excitonic states which are accumulated within the bulk of grains [54] or bound to impurities. This is supported by two facts: First, cross-sectional TEM analysis of a $\mathrm{MC}$ that is fabricated under the same conditions provides a granular structure of the investigated $\mathrm{ZnO} \mathrm{MC}$ with grain sizes ranging from 20 up to $120 \mathrm{~nm} \mathrm{[24].} \mathrm{Second,} \mathrm{the} \mathrm{slope}$ of the polariton blueshift $\Delta E(P)$ is, by a factor of about 6.3 , larger for $P<2 P_{\text {th }}$ than above and even larger, by a factor of 12.6, compared to the blueshift for $P>4 P_{\text {th }}$ (see Fig. 7). This can be explained by assuming an additional electronic background potential $\Delta E_{b}$, which may include localized states within a disorder potential or bound to impurities, as shown in Refs. [24,65]. Since the concentration of these electronic

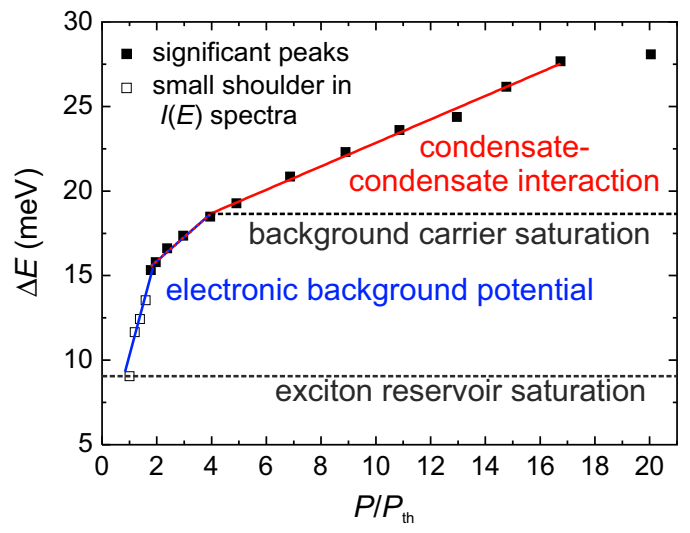

FIG. 7. Polariton blueshift $\Delta E$ as a function of the excitation power normalized to the condensation threshold for the observed $\mathrm{ZnO}$ MC. The red line corresponds to the polariton-polariton interaction, whereas the blue line represents the blueshift due to an additional electronic background potential, which starts to saturate at about $2 P_{\text {th }}$ and is presumably totally saturated for $P>4 P_{\text {th }}$. defects is finite, their contribution to the condensate blueshift saturates for a certain excitation power, or, rather, condensate density. Thus, the further blueshift for $P>4 P_{\text {th }}$ is restricted to condensate-condensate interaction.

We assume that the condensate blueshift for small excitation power $\Delta E\left(P<2 P_{\text {th }}\right)$ is primarily caused by its interaction with aluminum donor bound excitons $\left(D^{0}, X\right)$ and that $\Delta E$ scales linearly with its concentration. As mentioned in Sec. III, we suppose a depletion of bound excitons at grain boundaries and thus an accumulation of them within the grain bulk [54]. According to the model described in Ref. [54], the grain boundaries act like two back-to-back Schottky barriers, and the carrier flow between grains is driven by thermionic emission over the Schottky barrier. In general, the average height and width of these barriers can be determined from the temperature-dependent evolution of the Hall mobility. Unfortunately, this was not possible for our MC due to low current values, below the resolution limit of $1 \mathrm{nA}$, for temperatures below $200 \mathrm{~K}$, caused by the small cavity thickness of about $100 \mathrm{~nm}$, as well as due to strong inhomogeneities of the current density, which may be caused by the cavity thickness gradient.

Assuming the mechanism of carrier depletion at grain boundaries is the dominant one for the effective electronic disorder potential, its correlation length $\xi_{V}$ is similar to the grain size with values between 20 and $120 \mathrm{~nm}$. This is about two orders of magnitude below the condensate size $L_{c}$, limited by the size of the pump spot and thus even lower than the assumed correlation length for photonic disorder of about $370 \mathrm{~nm}$. Consequently, a trapping of the entire condensate within a minimum of the disorder potential can be excluded. We rather suppose that the disorder potential causes condensate density fluctuations and thus phase fluctuations due to the interplay of disorder and the nonequilibrium nature of the polariton condensate.

\section{APPENDIX C: DETAILS OF THE MODEL}

A phenomenological description of the dynamics of the macroscopic polariton condensate wave function $\Psi(\vec{x}, t)$ is given by an extended Gross-Pitaevskii equation (eGPE) $[47,48]$,

$$
\begin{aligned}
i \hbar \partial_{t} \Psi= & \left(-\frac{\hbar^{2}}{2 m} \vec{\nabla}^{2}+V(\vec{x})+U|\Psi|^{2}\right) \Psi \\
& +i\left(R(\vec{x})-\Gamma|\Psi|^{2}\right) \Psi .
\end{aligned}
$$

The first part of the right-hand side is the ordinary equilibrium GPE, with $m$ being the effective polariton mass of the lower polariton branch, $V(\vec{x})$ being the external potential, and $U$ being the repulsive on-site interaction potential. The second part models phenomenologically the gain and loss of condensed polaritons. Here, $R(\vec{x})$ describes the linear part of gain and loss, and the nonlinearity implements a density-dependent gain saturation, with $\Gamma$ being the gain depletion parameter. This provides a simplified description of the gain process from a reservoir, for example, relaxation of high-momentum polaritons generated by incoherent excitation with an external laser beam, and the condensate decay due to its finite lifetime. Since the noncondensed polaritons have a short lifetime compared to the lifetime of the condensate, we can 
safely neglect their diffusion processes and relate the spatial extension of the reservoir to the Gaussian excitation profile of the laser beam. Then,

$$
R(\vec{x})=\hbar \gamma_{\mathrm{c}}\left(\frac{P}{P_{\mathrm{th}}} e^{-\vec{x}^{2} / \xi_{P}^{2}}-1\right),
$$

with the decay rate $\gamma_{\mathrm{c}}=1 / \tau$, where $\tau$ is the condensate lifetime, and $\xi_{P}$ being the waist size of the laser beam. The parameter $P / P_{\text {th }}$ is the excitation power normalized by its value at the threshold at which condensation is observed first. The disorder landscape is incorporated by the external potential $V(\vec{x})$. We use a $\delta$-correlated Gaussian-distributed quenched disorder with vanishing mean value and variance,

$$
\langle\langle V(\vec{x})\rangle\rangle=0, \quad\langle\langle V(\vec{x}) V(\vec{y})\rangle\rangle=V_{0}^{2} \xi_{V}^{2} \delta(\vec{x}-\vec{y}),
$$

respectively. The average disorder strength is given by $V_{0}$, and its characteristic length is denoted by $\xi_{V}$.

In the case of a spatially homogeneous excitation, i.e., $\xi_{\mathrm{P}} \rightarrow$ $\infty$, our model (C1) was first suggested in Ref. [48]. In contrast to Ref. [47], we do not consider the dynamics of the reservoir polaritons explicitly. However, the latter can be eliminated [3] for the typical case that the characteristic relaxation rate of the reservoir is much faster than the condensate decay rate $[3,50]$. Then, an expansion to leading order in condensate density over reservoir density results in the eGPE (C1). We note that a different theoretical approach may be suitable for describing propagation of a polariton BEC in a disorder-free environment $[66,67]$, which is not the aim of our work.

In the following we will discuss the model (C1). The mean condensate density $n_{0} \equiv \frac{1}{\Omega_{\mathrm{c}}} \int_{\Omega_{\mathrm{c}}}|\Psi(\vec{x})|^{2}$ is found by averaging the second term on the right-hand side of Eq. (C1) over the condensate area $\Omega_{\mathrm{c}} \approx \pi \xi_{\mathrm{P}}^{2}$ and then demanding a balance of gain and loss,

$$
n_{0} \approx \frac{\hbar \gamma_{\mathrm{c}}}{\Gamma}\left(\frac{P}{P_{\mathrm{th}}}-1\right) .
$$

Since the interaction term in Eq. (C1) is proportional to the density, we find an energy blueshift $n_{0} U$. The healing length is extracted by comparing the kinetic-energy term with the interaction term in Eq. (C1),

$$
\xi \equiv \sqrt{\frac{\hbar^{2} / 2 m}{n_{0} U}} .
$$

Let us understand its physical relevance: For example, we assume a region in which the condensate has to vanish, $\Psi=0$, still remains unperturbed everywhere else. Then, the healing length is the distance over which the condensate density changes from zero to $n_{0}$.

A dimensionless eGPE (C1) takes the form

$$
i \partial_{t} \psi=\left[-\nabla^{2}+\vartheta(\vec{x})+|\psi|^{2}\right] \psi+i \alpha\left[g_{\mathrm{R}}(\vec{x})-|\psi|^{2}\right] \psi,
$$

where density, length, energy, and time are measured in units of $n_{0}, \xi, n_{0} U$, and $\hbar / n_{0} U$, respectively. The "nonequilibrium" parameter $\alpha$ and the dimensionless reservoir function $g_{\mathrm{R}}$ are defined in Eqs. (C8) and (C9), respectively. With $\psi(\vec{x}, t) \equiv$ $\Psi(\vec{r}, t) / \sqrt{n_{0}}$ we denote the dimensionless wave function, and $\vartheta(\vec{x})=V(\vec{x}) / n_{0} U$ is the disorder potential relative to the blueshift, with

$$
\langle\langle\vartheta(\vec{x})\rangle\rangle=0, \quad\langle\langle\vartheta(\vec{x}) \vartheta(\vec{y})\rangle\rangle=\kappa^{2} \delta(\vec{x}-\vec{y}) .
$$

We have introduced two important dimensionless parameters, namely, an effective disorder strength and a nonequilibrium parameter,

$$
\kappa \equiv \frac{\xi_{V} V_{0}}{\xi n_{0} U}, \quad \alpha \equiv \frac{\Gamma}{U}
$$

The first parameter $\kappa$ is also obtained by coarse graining the random disorder potential up to the healing length (assuming $\left.\xi_{V} \ll \xi\right)$. This process renormalizes the disorder strength by a factor $1 / \sqrt{\left(\xi / \xi_{V}\right)^{2}}$. Then, the value $\xi_{V} V_{0} / \xi$ is compared to the blueshift $n_{0} U$. The second parameter $\alpha$ implements the nonequilibrium nature of polaritons. In the limit $\alpha \rightarrow 0$ (keeping $n_{0}$ constant) Eq. (C6) reduces to the equilibrium GPE, whereas for $\alpha \rightarrow \infty$ the condensate is totally dominated by gain and loss. The rescaled reservoir function yields

$$
g_{\mathrm{R}}(\vec{x})=\frac{\left(P / P_{\mathrm{th}}\right) e^{-x^{2} / x_{P}^{2}}-1}{P / P_{\mathrm{th}}-1},
$$

with $x_{\mathrm{P}} \equiv \xi_{P} / \xi$. For a steady-state solution (single-mode condensate) we make the ansatz

$$
\psi(\vec{x}, t)=\psi(\vec{x}) e^{-i \omega t}=\sqrt{n(\vec{x})} e^{i \phi(\vec{x})-i \omega t},
$$

where $\hbar \omega$ is the condensate energy.

We emphasize that both blueshift and healing length depend on the excitation power $P$ via $n_{0}$. Thus, $\kappa$ and $x_{P}$ depend on $P$, too. For our analysis it is useful to identify energy and length scale which are excitation power independent, namely, the linewidth energy $\hbar \gamma_{\mathrm{c}}$ and the quantum correlation length $l_{\gamma} \equiv \sqrt{\hbar / 2 m \gamma_{\mathrm{c}}}$ (a nonequilibrium analogon of the thermal de Broglie wavelength) [25], so that $\kappa$ and $x_{P}$ become functions of $\alpha, P / P_{\text {th }}$, and sample parameters (see Table I).

\section{APPENDIX D: NUMERICAL SIMULATIONS AND COMPARISON WITH THE EXPERIMENT}

Numerical simulations. Computing the condensate wave function by solving the eGPE (C1) allows us to extract the real- and $k$-space intensity distribution. We define the $k$-space intensity distribution according to

$$
I_{P}(\vec{k}) \equiv \gamma_{\mathrm{c}} n_{0}|\psi(\vec{k})|^{2},
$$

where the momentum-space wave function is defined via a two-dimensional discrete Fourier transform $\psi\left(\vec{k}_{j}\right)=$ $\left(1 / N^{2}\right) \sum_{\vec{x}_{i}} \psi\left(\vec{x}_{i}\right) e^{-i \vec{k}_{j} \vec{x}_{i}}$, with $\vec{x}_{i}, \vec{k}_{j}$ being elements of a discrete lattice with $N$ lattice points in each spatial direction, such that $i, j=1, \ldots, N^{2}$. We choose an appropriate set of simulation parameters extracted from the experiment (see Table I) and solve Eq. (C6) numerically. To this end we look for a steady-state solution [see Eq. (C10)] by solving the time evolution of the discretized wave function $\psi\left(\vec{x}_{i}, t\right)$. The latter is defined on a real-space square lattice with spacing $a=$ $\xi$. We employ a variable-order Adams-Bashforth-Moulton algorithm [68] to obtain the time evolution. First, we compute the steady-state solution of the disorder-free system, $\vartheta=0$. Then, we choose independent Gaussian-distributed variables of vanishing mean and variance $\kappa^{2}$ for each lattice site and calculate the steady-state solution of the disordered system. The time evolution of the disordered system is started with the disorder-free solution as the initial condition. For each 


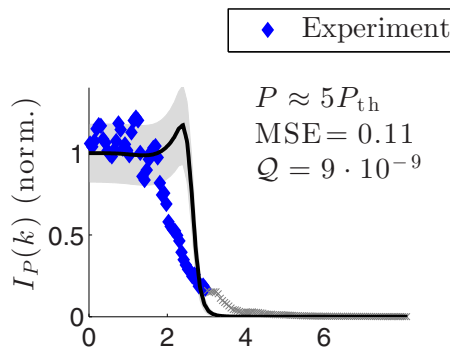

Theory
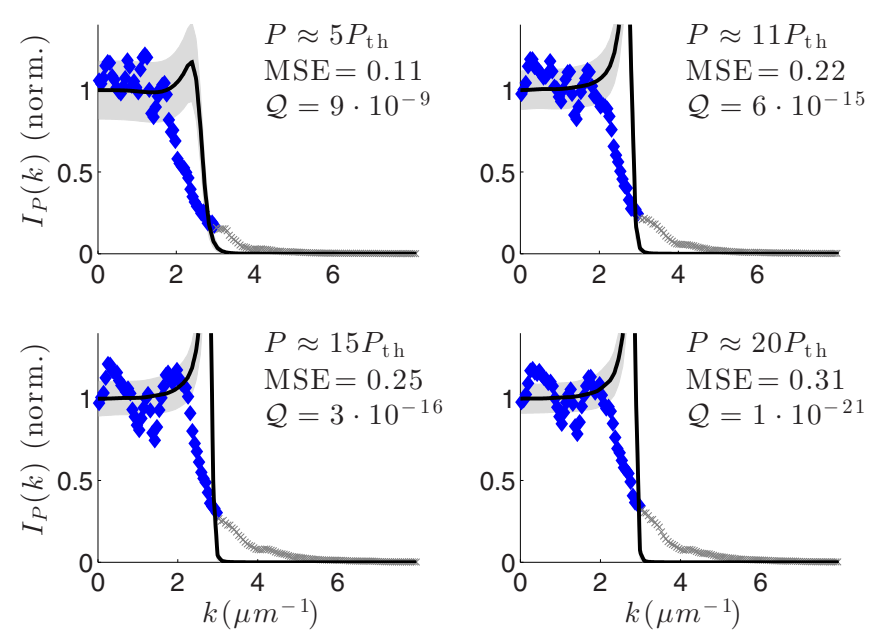

FIG. 8. Experimental $I_{P}(k)$ distribution (blue diamonds) compared to numerical simulations of scenario I for increasing excitation power $P$. The black solid lines depict the expectation value, whereas the gray band indicates the standard deviation. For high momenta we have excluded systematically biased data (gray crosses). For each plot the mean-square error (MSE) and the goodness-of-fit value $\mathcal{Q}$ are computed. Simulation parameters: $\alpha=0.5, \xi_{\mathrm{V}} V_{0} / l_{\mathrm{c}} \hbar \gamma_{\mathrm{c}}=0.4$, $\xi_{P} / l_{\mathrm{c}}=3$. These correspond to $\mathcal{L}_{\mathrm{n}} / L_{c} \approx 1.5, \mathcal{L}_{\phi} / L_{c} \approx 13, \mathcal{L}_{s} / L_{c} \approx 26$.

disorder realization the discretized two-dimensional wave function $\psi\left(\vec{x}_{i}\right)$ of the steady state is extracted and then Fourier transformed in order to compute the two-dimensional $k$-space intensity $I_{P}\left(\vec{k}_{j}\right)$. Finally, we average over all disorder realizations and compute the expectation value and variance,

$$
\begin{gathered}
\mu_{P}(k) \equiv\left\langle\left\langle I_{P}(\vec{k})\right\rangle\right\rangle /\left\langle\left\langle I_{P}(0)\right\rangle\right\rangle, \\
\sigma_{P}^{2}(k) \equiv\left\langle\left\langle\left(I_{P}(\vec{k})-\left\langle\left\langle I_{P}(\vec{k})\right\rangle\right)^{2}\right\rangle\right\rangle /\left\langle\left\langle I_{P}(0)\right\rangle\right\rangle^{2},\right.
\end{gathered}
$$

respectively. Above, the brackets $\langle\langle\cdots\rangle\rangle$ denote an average with respect to disorder, and we have normalized the mean and variance by the expectation value of the intensity at $k=0$. Since the excitation profile (C9) is radial symmetric, Eqs. (D2) and (D3) are radial symmetric, too, assuming a sufficiently large number of disorder realizations.

Comparison with the experiment. The numerically obtained mean and variance of the $k$-space intensity can be compared with the experimental data denoted by $I_{\mathrm{ex}}\left(k_{x}\right)$ here (see Sec. III). We note that these measurements represent a line cut along an axis (e.g., the $x$ axis) of the two-dimensional intensity distribution and are measured for one disorder configuration determined by the disorder of the sample. We perform a spatial averaging step by symmetrizing the experimentally obtained intensity: $I_{\mathrm{ex}}\left(k_{x}\right) \rightarrow\left[I_{\mathrm{ex}}\left(k_{x}\right)+I_{\mathrm{ex}}\left(-k_{x}\right)\right] / 2$ and $k_{x} \geqslant 0$. In order to quantify the agreement between experiment and theory we introduce the $\chi^{2}$ value [68],

$$
\chi_{P}^{2}=\sum_{k_{j} \geqslant 0}\left(\frac{I_{\mathrm{ex}}\left(k_{j}\right) / a-\mu_{P}\left(b k_{j}\right)}{\sigma_{P}\left(b k_{j}\right)}\right)^{2} .
$$

Since the condensate density $n_{0}$ and the healing length $\xi$ are hard to extract experimentally, we use two scaling parameters $(a, b)$ instead. Both are determined by a least-squares fitting procedure [68].

In order to estimate the goodness of fit [68] we extract the complement of the $\chi^{2}$-probability distribution function $F_{\chi^{2}}$, denoted by $\mathcal{Q}=1-F_{\chi^{2}}\left(\chi_{P}^{2}\right)$, which is the probability that the simulations agree with the experimental data. If $\mathcal{Q} \ll 1$, the apparent discrepancies of model and data are unlikely to be random fluctuations, and we conclude that the model is not specified correctly or that the fluctuation strength $\sigma_{P}$ is underestimated. On the other hand, if $\mathcal{Q} \sim 1$, we conclude that the model describes the data correctly. Finally, we define the mean-square error: $\operatorname{MSE} \equiv\left(1 / N^{2}\right) \sum_{k_{j}}\left[I_{\mathrm{ex}}\left(k_{j}\right) / a-\right.$ $\left.\mu_{P}\left(k_{j}\right)\right]^{2}$, which is a measure of how well the data match the simulated intensity distribution. The comparison of the experimental data and the numeric simulations of scenario I is shown in Fig. 8, and the comparison with simulations of scenario II is shown and discussed in Sec. IV (Fig. 6).
[1] J. Kasprzak, M. Richard, S. Kundermann, A. Baas, P. Jeambrun, J. M. J. Keeling, F. M. Marchetti, M. H. Szymańska, R. André, J. L. Staehli, V. Savona, P. B. Littlewood, B. Deveaud, and L. S. Dang, Nature (London) 443, 409 (2006).

[2] R. Balili, V. Hartwell, D. Snoke, L. Pfeiffer, and K. West, Science 316, 1007 (2007).

[3] I. Carusotto and C. Ciuti, Rev. Mod. Phys. 85, 299 (2013).

[4] H. Deng, H. Haug, and Y. Yamamoto, Rev. Mod. Phys. 82, 1489 (2010).

[5] A. Amo, D. Sanvitto, F. P. Laussy, D. Ballarini, E. d. Valle, M. D. Martín, A. Lemaître, J. Bloch, D. N. Krizhanovskii, M. S. Skolnick, C. Tejedor, and L. Viña, Nature (London) 457, 291 (2009).

[6] A. Amo, J. Lefrère, S. Pigeon, C. Adrados, C. Ciuti, I. Carusotto, R. Houdré, E. Giacobino, and A. Bramati, Nat. Phys. 5, 805 (2009).
[7] D. Sanvitto, F. M. Marchetti, M. H. Szymańska, G. Tosi, M. Baudisch, F. P. Laussy, D. N. Krizhanovskii, M. S. Skolnick, L. Marrucci, A. Lemaître, J. Bloch, C. Tejedor, and L. Viña, Nat. Phys. 6, 527 (2010).

[8] K. G. Lagoudakis, M. Wouters, M. Richard, A. Baas, I. Carusotto, R. André, L. S. Dang, and B. Deveaud-Plédran, Nat. Phys. 4, 706 (2008).

[9] J. J. Baumberg, P. G. Savvidis, R. M. Stevenson, A. I. Tartakovskii, M. S. Skolnick, D. M. Whittaker, and J. S. Roberts, Phys. Rev. B 62, R16247 (2000).

[10] C. Schneider, A. Rahimi-Iman, N. Y. Kim, J. Fischer, I. G. Savenko, M. Amthor, M. Lermer, A. Wolf, L. Worschech, V. D. Kulakovskii, I. A. Shelykh, M. Kamp, S. Reitzenstein, A. Forchel, Y. Yamamoto, and S. Höfling, Nature (London) 497, 348 (2013).

[11] P. Bhattacharya, T. Frost, S. Deshpande, M. Z. Baten, A. Hazari, and A. Das, Phys. Rev. Lett. 112, 236802 (2014). 
[12] D. Bajoni, E. Semenova, A. Lemaître, S. Bouchoule, E. Wertz, P. Senellart, S. Barbay, R. Kuszelewicz, and J. Bloch, Phys. Rev. Lett. 101, 266402 (2008).

[13] M. Steger, C. Gautham, B. Nelsen, D. Snoke, L. Pfeiffer, and K. West, Appl. Phys. Lett. 101, 131104 (2012).

[14] D. Ballarini, M. De. Giorgi, E. Cancellieri, R. Houdré, E. Giacobino, R. Cingolani, A. Bramati, G. Gigli, and D. Sanvitto, Nat. Commun. 4, 1778 (2013).

[15] C. Antón, T. C. H. Liew, J. Cuadra, M. D. Martín, P. S. Eldridge, Z. Hatzopoulos, G. Stavrinidis, P. G. Savvidis, and L. Viña, Phys. Rev. B 88, 245307 (2013).

[16] C. Sturm, D. Tanese, H. S. Nguyen, H. Flayac, E. Galopin, A. Lemaître, I. Sagnes, D. Solnyshkov, A. Amo, G. Malpuech, and J. Bloch, Nat. Commun. 5 (2014).

[17] S. Christopoulos, G. Baldassarri Höger von Högersthal, A. J. D. Grundy, P. G. Lagoudakis, A. V. Kavokin, J. J. Baumberg, G. Christmann, R. Butté, E. Feltin, J.-F. Carlin, and N. Grandjean, Phys. Rev. Lett. 98, 126405 (2007).

[18] G. Christmann, R. Butté, E. Feltin, J.-F. Carlin, and N. Grandjean, Appl. Phys. Lett. 93, 051102 (2008).

[19] K. S. Daskalakis, P. S. Eldridge, G. Christmann, E. Trichas, R. Murray, E. Iliopoulos, E. Monroy, N. T. Pelekanos, J. J. Baumberg, and P. G. Savvidis, Appl. Phys. Lett. 102, 101113 (2013).

[20] T.-C. Lu, Y.-Y. Lai, Y.-P. Lan, S.-W. Huang, J.-R. Chen, Y.-C. Wu, W.-F. Hsieh, and H. Deng, Opt. Express 20, 5530 (2012).

[21] F. Li, L. Orosz, O. Kamoun, S. Bouchoule, C. Brimont, P. Disseix, T. Guillet, X. Lafosse, M. Leroux, J. Leymarie, M. Mexis, M. Mihailovic, G. Patriarche, F. Réveret, D. Solnyshkov, J. Zúñiga-Pérez, and G. Malpuech, Phys. Rev. Lett. 110, 196406 (2013).

[22] Y.-Y. Lai, Y.-P. Lan, and T.-C. Lu, Appl. Phys. Express 5, 082801 (2012).

[23] J. D. Plumhof, T. Stöferle, L. Mai, U. Scherf, and R. F. Mahrt, Nat. Mater. 13, 247 (2014).

[24] H. Franke, C. Sturm, R. Schmidt-Grund, G. Wagner, and M. Grundmann, New J. Phys. 14, 013037 (2012).

[25] A. Trichet, E. Durupt, F. Médard, S. Datta, A. Minguzzi, and M. Richard, Phys. Rev. B 88, 121407 (2013).

[26] G. Roumpos, M. Lohse, W. H. Nitsche, J. Keeling, M. H. Szymanska, P. B. Littlewood, A. Löffler, S. Höfling, L. Worschech, A. Forchel, and Y. Yamamoto, Proc. Natl. Acad. Sci. USA 109, 6467 (2012).

[27] A. Chiocchetta and I. Carusotto, Europhys. Lett. 102, 67007 (2013).

[28] R. Spano, J. Cuadra, G. Tosi, C. Antón, C. A. Lingg, D. Sanvitto, M. D. Martín, L. Viña, P. R. Eastham, M. van der Poel, and J. M. Hvam, New J. Phys. 14, 075018 (2012).

[29] R. Spano, J. Cuadra, C. Lingg, D. Sanvitto, M. D. Martín, P. R. Eastham, M. van der Poel, J. M. Hvam, and L. Viña, Opt. Express 21, 10792 (2013).

[30] M. Wouters and I. Carusotto, Phys. Rev. Lett. 105, 020602 (2010).

[31] J. Keeling, Phys. Rev. Lett. 107, 080402 (2011).

[32] L. M. Sieberer, S. D. Huber, E. Altman, and S. Diehl, Phys. Rev. Lett. 110, 195301 (2013).

[33] L. M. Sieberer, S. D. Huber, E. Altman, and S. Diehl, Phys. Rev. B 89, 134310 (2014).

[34] U. C. Täuber and S. Diehl, Phys. Rev. X 4, 021010 (2014).
[35] E. Altman, L. M. Sieberer, L. Chen, S. Diehl, and J. Toner, Phys. Rev. X 5, 011017 (2015).

[36] A. Janot, T. Hyart, P. R. Eastham, and B. Rosenow, Phys. Rev. Lett. 111, 230403 (2013).

[37] T. Nattermann and V. L. Pokrovsky, Phys. Rev. Lett. 100, 060402 (2008).

[38] G. M. Falco, T. Nattermann, and V. L. Pokrovsky, Phys. Rev. B 80, 104515 (2009).

[39] G. Malpuech, D. D. Solnyshkov, H. Ouerdane, M. M. Glazov, and I. Shelykh, Phys. Rev. Lett. 98, 206402 (2007).

[40] F. Manni, K. G. Lagoudakis, B. Pietka, L. Fontanesi, M. Wouters, V. Savona, R. André, and B. Deveaud-Plédran, Phys. Rev. Lett. 106, 176401 (2011).

[41] P. Stępnicki and M. Matuszewski, Phys. Rev. A 88, 033626 (2013).

[42] M. Richard, J. Kasprzak, R. Romestain, R. André, and L. S. Dang, Phys. Rev. Lett. 94, 187401 (2005).

[43] A. Baas, K. G. Lagoudakis, M. Richard, R. André, L. S. Dang, and B. Deveaud-Plédran, Phys. Rev. Lett. 100, 170401 (2008).

[44] D. N. Krizhanovskii, K. G. Lagoudakis, M. Wouters, B. Pietka, R. A. Bradley, K. Guda, D. M. Whittaker, M. S. Skolnick, B. Deveaud-Plédran, M. Richard, R. André, and L. S. Dang, Phys. Rev. B 80, 045317 (2009).

[45] M. Wouters, Phys. Rev. B 77, 121302 (2008).

[46] P. R. Eastham, Phys. Rev. B 78, 035319 (2008).

[47] M. Wouters and I. Carusotto, Phys. Rev. Lett. 99, 140402 (2007).

[48] J. Keeling and N. G. Berloff, Phys. Rev. Lett. 100, 250401 (2008).

[49] Y. Imry and S.-K. Ma, Phys. Rev. Lett. 35, 1399 (1975).

[50] M. Wouters, I. Carusotto, and C. Ciuti, Phys. Rev. B 77, 115340 (2008).

[51] E. Wertz, L. Ferrier, D. Solnyshkov, R. Johne, D. Sanvitto, A. Lemaître, I. Sagnes, R. Grousson, A. V. Kavokin, P. Senellart, G. Malpuech, and J. Bloch, Nat. Phys. 6, 860 (2010).

[52] C. Antón, T. C. H. Liew, G. Tosi, M. D. Martín, T. Gao, Z. Hatzopoulos, P. S. Eldridge, P. G. Savvidis, and L. Viña, Phys. Rev. B 88, 035313 (2013).

[53] Aluminum is a common donor in $\mathrm{ZnO}$ films, and as a component of the sapphire substrate and Bragg mirror layers it can easily diffuse into the $\mathrm{ZnO}$ cavity during the annealing process at high temperatures of $T \approx 900^{\circ}$.

[54] J. W. Orton and M. J. Powell, Rep. Prog. Phys. 43, 1263 (1980).

[55] See Supplemental Material at http://link.aps.org/supplemental/ 10.1103/PhysRevB.93.064203 for details of the experimental determination of the condensation threshold density $P$ th as well as for experimental limitations for the determination of the coherence time.

[56] D. N. Krizhanovskii, D. Sanvitto, A. P. D. Love, M. S. Skolnick, D. M. Whittaker, and J. S. Roberts, Phys. Rev. Lett. 97, 097402 (2006).

[57] Note that the superposition of photons originating from polaritons with equal wave vectors would be sufficient to determine their coherence time. However, we decided to superimpose states with opposite $k$ values since, in the case of a propagating condensate, additional spatial coherence would be required to induce the observed interference pattern. This can be attributed to the fact that the emitted photons with different $k$ values may originate from different regions of the sample. This method 
does not change much the measured coherence time since reduced spatial correlations should only reduce the maximum value for the measured visibility (for zero temporal delay) but would not affect its general behavior with increasing temporal delay.

[58] B. E. A. Saleh and M. C. Teich, Fundamentals of Photonics, 2nd ed. (John Wiley \& Sons, Inc., New Jersey, 2007).

[59] D. Porras and C. Tejedor, Phys. Rev. B 67, 161310 (2003).

[60] The nonequilibrium parameter $\alpha$ can be extracted by the linewidth divided by the derivative of the condensate blueshift with respect to excitation power, $\alpha=\hbar \gamma_{\mathrm{c}} /\left[d \Delta E / d\left(P / P_{\mathrm{th}}\right)\right]$. Within the error bounds we chose $\alpha=7$ in order to reproduce the experimental data.

[61] The shoulder in the experimental data at $k=3, \ldots, 4 \mu \mathrm{m}^{-1}$ is visible for all excitation powers, and its shape and magnitude are independent of the power. Therefore, we can conclude that this shoulder is caused by the residual effect and does not belong to the condensate emission. Different options are possible, e.g., the emission of uncondensed polaritons occupying the lower polariton branch or artifacts from the experimental setup, e.g., transmission fluctuations from the microscope objective (see Sec. III).

[62] L. Orosz, F. Réveret, F. Médard, P. Disseix, J. Leymarie, M. Mihailovic, D. Solnyshkov, G. Malpuech, J. Zúñiga-Pérez, F. Semond, M. Leroux, S. Bouchoule, X. Lafosse, M. Mexis, C. Brimont, and T. Guillet, Phys. Rev. B 85, 121201 (2012).

[63] V. Savona, J. Phys. Condens. Matter 19, 295208 (2007).

[64] G. Nardin, K. G. Lagoudakis, M. Wouters, M. Richard, A. Baas, R. André, L. S. Dang, B. Pietka, and B. Deveaud-Plédran, Phys. Rev. Lett. 103, 256402 (2009).

[65] H. Franke, Ph.D. thesis, Universität Leipzig, 2012, http://nbnresolving.de/urn:nbn:de:bsz:15-qucosa-98174.

[66] E. Wertz, A. Amo, D. D. Solnyshkov, L. Ferrier, T. C. H. Liew, D. Sanvitto, P. Senellart, I. Sagnes, A. Lemaître, A. V. Kavokin, G. Malpuech, and J. Bloch, Phys. Rev. Lett. 109, 216404 (2012).

[67] D. D. Solnyshkov, H. Terças, K. Dini, and G. Malpuech, Phys. Rev. A 89, 033626 (2014).

[68] W. H. Press, S. A. Teukolsky, W. T. Vetterling, and B. P. Flannery, Numerical Recipes: The Art of Scientific Computing (Cambridge University Press, Cambridge, 2007). 